\title{
Using Multiple \\ Unmanned Systems for a Site Security Task
}

\section{Defense Transformation and Net- Centric Systems 2009}

\author{
Matthew O. Anderson \\ Curtis W. Nielsen \\ Mark C. McKay \\ Derek C. Wadsworth
}

The INL is a

U.S. Department of Energy

National Laboratory

operated by

Battelle Energy Alliance

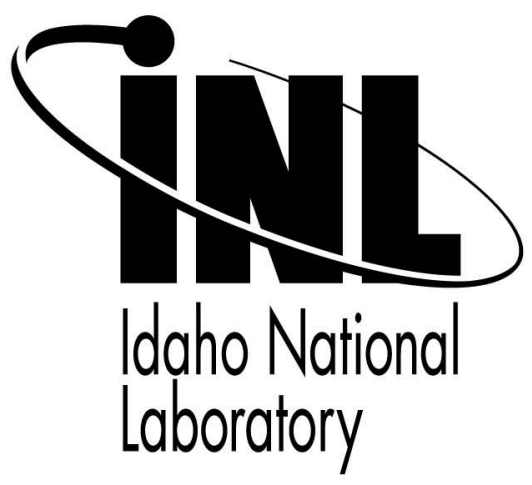

This is a preprint of a paper intended for publication in a journal or proceedings. Since changes may be made before publication, this preprint should not be cited or reproduced without permission of the author. This document was prepared as an account of work sponsored by an agency of the United States Government. Neither the United States Government nor any agency thereof, or any of their employees, makes any warranty, expressed or implied, or assumes any legal liability or responsibility for any third party's use, or the results of such use, of any information, apparatus, product or process disclosed in this report, or represents that its use by such third party would not infringe privately owned rights. The views expressed in this paper are not necessarily those of the United States Government or the sponsoring agency. 


\title{
Using Multiple Unmanned Systems for a Site Security Task
}

\author{
Matthew O. Anderson ${ }^{\mathrm{a}}$, Curtis W. Nielsen ${ }^{\mathrm{a}}$, Mark D. McKay ${ }^{\mathrm{a}}$, Derek C. Wadsworth, \\ Ryan C. Hruska ${ }^{a}$, and John A. Koudelka ${ }^{\mathrm{a}}$ \\ a'Idaho National Laboratory, P.O. Box 1625, Idaho Falls, ID, USA 83415-2220;
}

\begin{abstract}
Unmanned systems are often used to augment the ability of humans to perform challenging tasks. While the value of individual unmanned vehicles has been proven for a variety of tasks, it is less understood how multiple unmanned systems should be used together to accomplish larger missions such as site security. The purpose of this paper is to discuss efforts by researchers at the Idaho National Laboratory (INL) to explore the utility and practicality of operating multiple unmanned systems for a site security mission. This paper reviews the technology developed for a multi-agent mission and summarizes the lessons-learned from a technology demonstration.
\end{abstract}

Keywords: Unmanned Systems, Robotics, Multi-Agent, Usability Experiments

\section{INTRODUCTION}

Unmanned systems are often used to augment the ability of humans to perform challenging tasks. While the value of individual unmanned vehicles has been proven for a variety of individual tasks, it is less understood how multiple unmanned systems should be used together to accomplish larger missions such as site security. The purpose of this paper is to discuss efforts by researchers at the Idaho National Laboratory to explore the utility and practicality of operating multiple unmanned systems.

\section{BACKGROUND}

In July, 2006, INL security and Department of Energy (DOE) headquarters personnel attended a technology demonstration to witness first hand the potential security advantages offered by the use of lightweight, man-portable unmanned air vehicles (UAVs) capable of performing real-time mission planning and persistent surveillance. For the demonstration, there was one team attempting to infiltrate the main base while the other team was tasked to intercept the invaders before the main facility was breached. The demonstration was performed twice, once without additional technology and once with various sensors and an UAV. The UAV was used to cooperate with the ground security force by providing an eye in the sky and was tasked with identifying intruders, following and assessing the situation including simulated barrier explosions, and final battle damage as well as simulated kill assessments.

Without technology the invading team approached from two miles out, broke through a jersey-barrier (about 300 meters away from the main target), came through fences, a demilitarized zone, and stormed the fire station where observers were located.

In contrast, when the UAV and sensors were in use, the invading team was spotted as they were rendezvousing for the attack two miles out. Operators of the UAV were able to direct the defending team to appropriate locations and neutralize the simulated attack before the opposing team crossed the jersey-barriers.

This demonstration provided a good illustration of the potential use of cutting edge technology in a site security mission. There were, however, some significant lessons learned regarding the practicality of actually using the system. For example, the UAV had a camera that required constant manual attention in order to keep it focused on a particular place of interest, which was particularly challenging when the target to track was moving. Moreover, the UAV camera did not have geo-referenced tracking, and relied on the operator's recognition of landmarks in the video image in order to task the ground forces to the correct place. ${ }^{1}$ 
In addition to the operator challenges with the UAV, the system for visualizing the information from the unmanned vehicles and sensors was difficult for operators to follow. The information was visible in a typical closed-circuit television fashion where each panel from the various sensors and cameras was displayed separately and distributed over a large video wall. Throughout the exercise, observers had to be directed where to look next in order to follow the progression of the assault. Figure 1 shows the twelve screens that were used to visualize the simulated invasion.

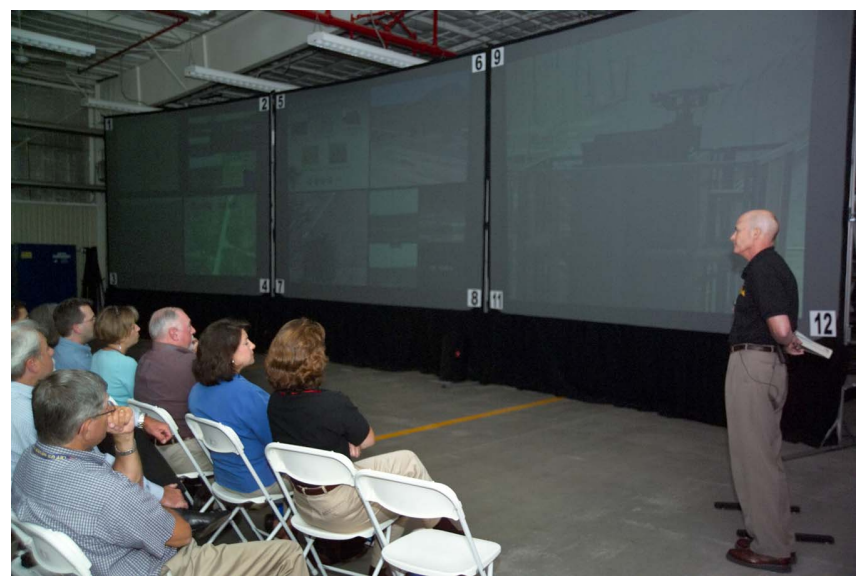

Figure 1. The video wall used to observe the progression of a simulated assault.

\section{IMPROVED TECHNOLOGY SCENARIO}

Based upon the lessons learned and experiences from the technology demonstration for site security, the highest technology gaps were identified, filled, and a new experiment was designed to show and evaluate an assortment of new technologies and new solutions. To that end, the overall scenario was the same as with the original technology demonstration with the exception that a suite of unmanned vehicles would be used as the primary means to intercept the invaders. In addition to the persistent surveillance, the capabilities of multiple smaller air vehicles and medium sized unmanned ground vehicles were used to address different aspects of the site security mission. Furthermore, since site security tasks involve defending a known site, there is no need to limit the solutions to approaches designed solely to work in unstructured, unknown environments. Rather the technology should be designed to take advantage of prior knowledge such as high-resolution imagery, LIDAR (light detection and ranging) data, terrain data, and GIS (geographic information systems) data, which can be used for the analysis of the most likely path of approach, optimal intercept paths, and a priori path planning for ground vehicles. Figure 2 shows the environment used for the demonstration which is located near the unmanned air vehicle runway at the INL facilities. ${ }^{1}$

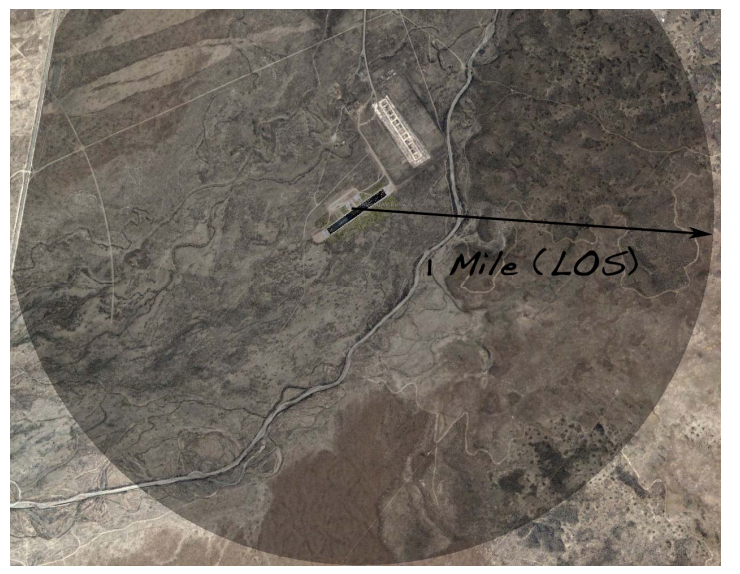

Figure 2. The environment and surveillance area used for the site security scenario. 


\section{TECHNOLOGY COMPONENTS}

Technology developed for this demonstration consisted of the following areas: prior knowledge of the site, unattended ground sensors, unmanned air vehicles, unmanned ground vehicles, and a common operating picture.

\subsection{Prior site knowledge}

Manned Flights over the experimental area were conducted to obtain high resolution imagery and LIDAR terrain data with an average posting every $30 \mathrm{~cm}$ and error bounds of $+/-5 \mathrm{~cm}$ vertical and $+/-11 \mathrm{~cm}$ horizontal. Figure 3 shows the quality of the LIDAR data (notice the detected power lines shown in red). Additionally, high-resolution $(7 \mathrm{~cm})$ imagery was also obtained to be used as the backdrop for the scenario (see discussion on common operating picture).

Prior to the demonstration the LIDAR and high resolution imagery were geo-referenced and used to compute likely paths of approach and paths of intercept given starting coordinates of a robot. This allowed the ground vehicle to be tasked immediately upon receipt of information regarding the position of an intruder.
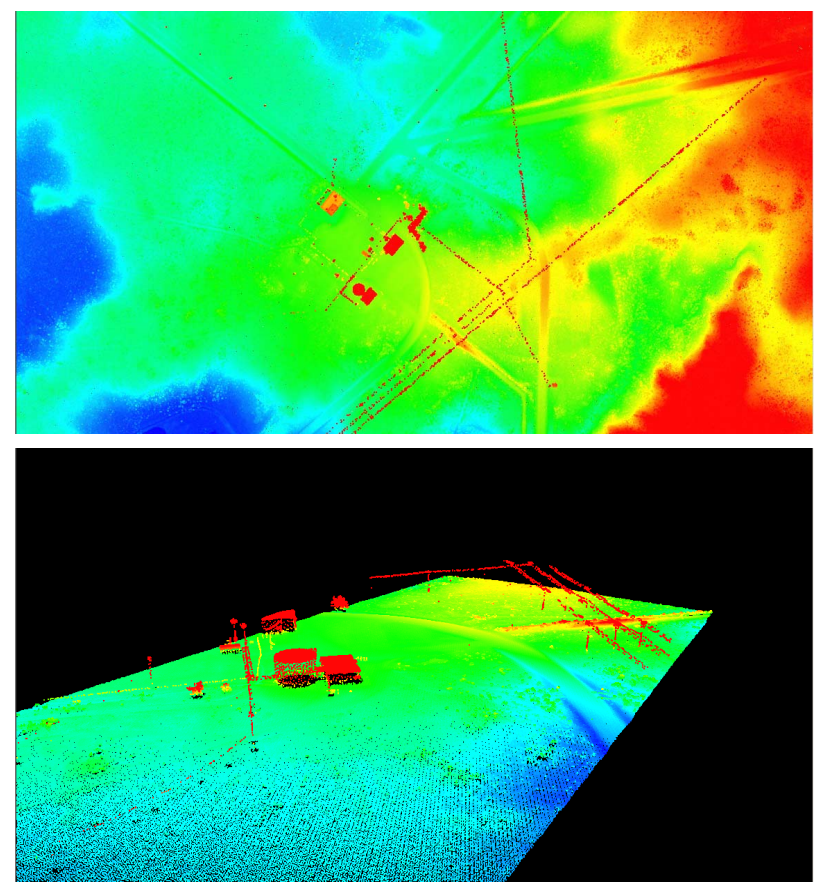

Figure 3. High resolution LIDAR data, blue represents lower elevation, red represents higher elevation.

\subsection{Unattended ground sensors.}

Sensors such as the Stabilized Panoramic Intruder Detection and Recognition System (SPIDER) and RADAR based unattended ground sensors provide initial intrusion detection. Also a Hyperspectral Unattended Ground Sensor (HUGS) that requires a specific target matrix defining how the targets are different from the background (i.e. people versus animals) allows for material identification. Other unattended ground sensors were identified that utilized acoustic, seismic, video, or a combination of each.

\subsection{Unmanned Air Vehicles}

Multiple UAVs were developed for this experiment to provide persistent surveillance, first response, and threat neutralization and confirmation capabilities.

\subsubsection{Persistent Surveillance}

A single 2-hour UAV system capable of deploying single or dual, gimbaled cameras with real-time imagery transmission to an operator control unit was developed to perform persistent surveillance. This design allowed persistent surveillance to continue with one gimbaled camera while the other gimbaled camera was employed for potential target investigation. The primary fixed wing platform is the Arcturus UAV T-15 airframe as shown in Figure 4 (see [1] for more details). The 
$\mathrm{T}-15$ is outfitted with a stabilized gimbaled video system weighing approximately $32 \mathrm{oz}$. with a 4.4 inch diameter turret as seen in Figure 5 (left side). This camera system is fully integrated with the T-15 autopilot allowing either manual control or automated "sensor point of interest" control that keeps the commanded Latitude / Longitude / Altitude point of interest in continuous view, while the UAV traverses the commanded flight path. Tracking software provided by Solution Engineering and Cloud Cap Technologies was used to task the camera system and the user interface is shown in Figure 5 (right side). This tracking software was designed to alleviate much of the human-in-the-loop target tracking that was observed during the 2006 security force on force demonstration.

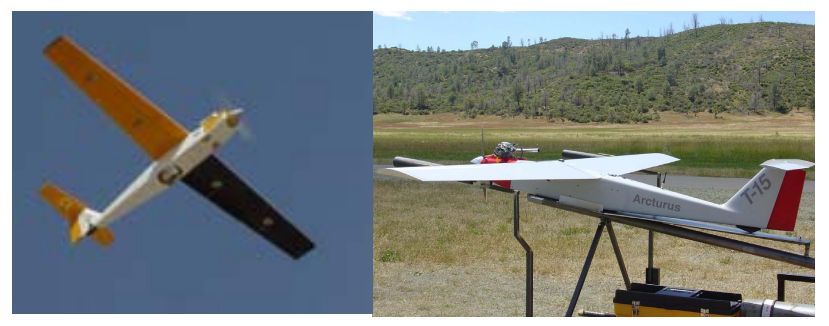

Figure 4. The Arcturus T-15 Unmanned Air Vehicle used in both demonstrations.

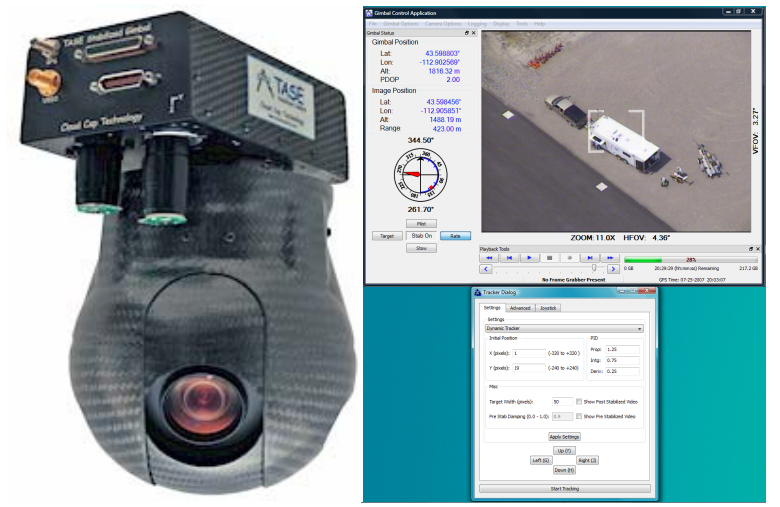

Figure 5. The camera system and tracking software integrated with the Arcturus T-15 UAV.

\subsubsection{First Response}

Multiple First Response Unmanned Air Vehicles (FRAVs) capable of low altitude flight, simultaneous target assessment, and dispersion algorithms for multiple target following were developed to perform first responder activities. Target coordinates and relevant information were conveyed to the operator control unit in real-time. The Magicc Lab at Brigham Young University developed the FRAVs (see Figure 6) with the following capabilities.

- Rapid deployment. A single operator can launch several systems without assistance using auto-take-off functionality. - Terrain following. Use on-board sensors and terrain data to maintain an altitude of approximately 30 meters above ground level. ${ }^{2,3}$

- Simultaneous arrival. Plan multiple flight paths so the air vehicles arrive simultaneously at the ground target with equal angular spacing. ${ }^{4}$

- Geo-location. Orbit the ground target and use on-board computer vision to estimate the air vehicles GPS location and disperse multiple air vehicles as necessary to track multiple targets. ${ }^{5}$ 


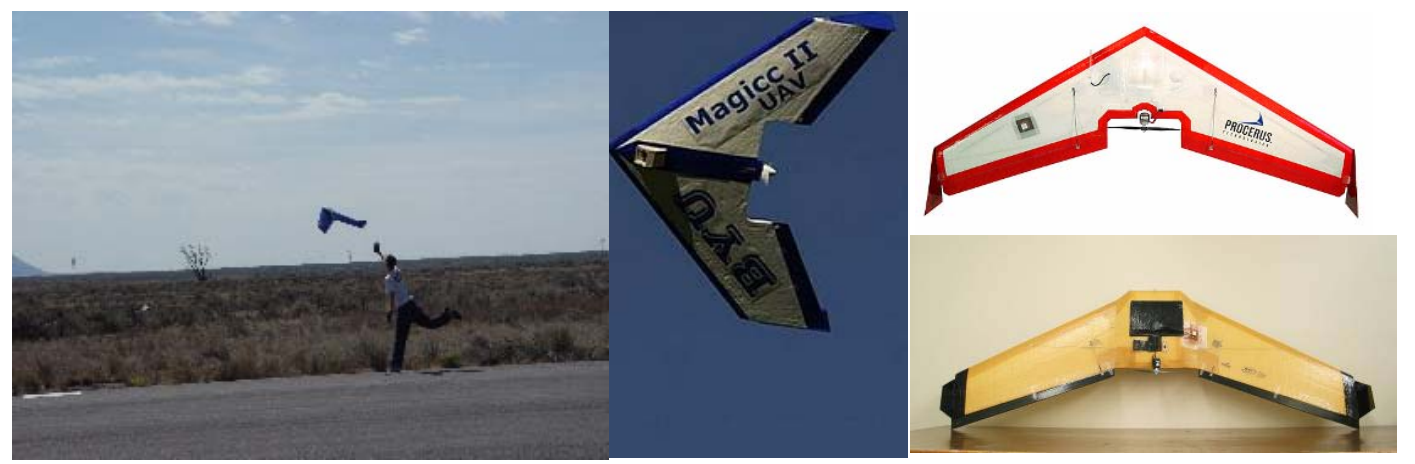

Figure 6. First Responder Air Vehicles developed by the BYU Magicc Lab.

\subsubsection{Threat neutralization and confirmation}

A manual radio-controlled rotary platform suitable for manual flight, manual geo-referenced video tracking, manual threat neutralization, and visual confirmation was developed by Neural Robotics Inc. Both electric (10 minute mission) and gas (30 minute mission) powered platforms were developed. Figure 7 shows the electric (left side) and gas (right side) platforms.

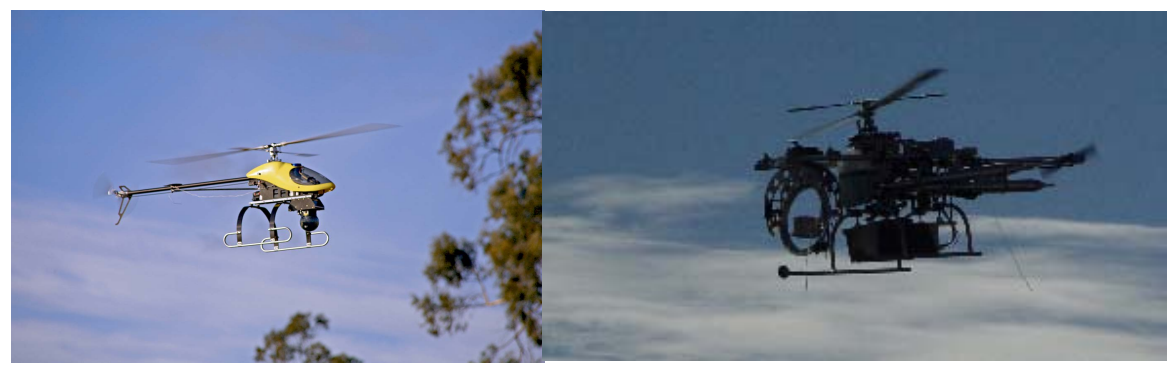

Figure 7. Rotary-winged aircraft used for threat neutralization and confirmation.

\subsection{Unmanned Ground Vehicles}

Multiple UGVs were developed for this experiment to provide robotic capabilities ranging from teleoperation to fully autonomous.

\subsubsection{Robotic Intelligent Node (RINO)}

Medium sized unmanned ground vehicles for ground-based interception were developed using a commercial off-theshelf Yamaha Rhino ATV and an electric GEM car. The RINO platform can be remotely driven via a wireless radio modem or can be operated through a robot intelligence kernel (RIK) developed previously at the INL. ${ }^{6}$ The RINO includes a global positioning system, two SICK laser scanners, an inertial navigation unit, and radar obstacle detection system and is capable of operating at speeds up to $30 \mathrm{mph}$. Figure 8 shows the RINO used for the demonstration. 


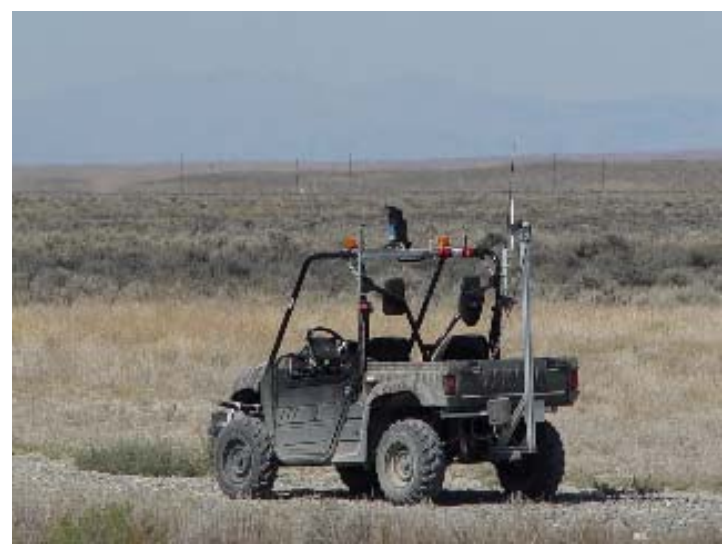

Figure 8. The RINO developed at the INL.

\subsubsection{Electric Robotic Intelligent Node (E-RINO)}

The E-RINO is built on a GEM Car platform and provides all of the functionality of the RINO with exception of range and duration. E-RINO is capable of speeds up to $25 \mathrm{mph}$ at a range of approximately 20 miles. Figure 9 shows the ERINO used for the demonstration. One clear advantage of the E-RINO is the absence of an internal combustion engine and the very low noise signature. For this reason, the E-RINO was used as the intruder for the demonstration.

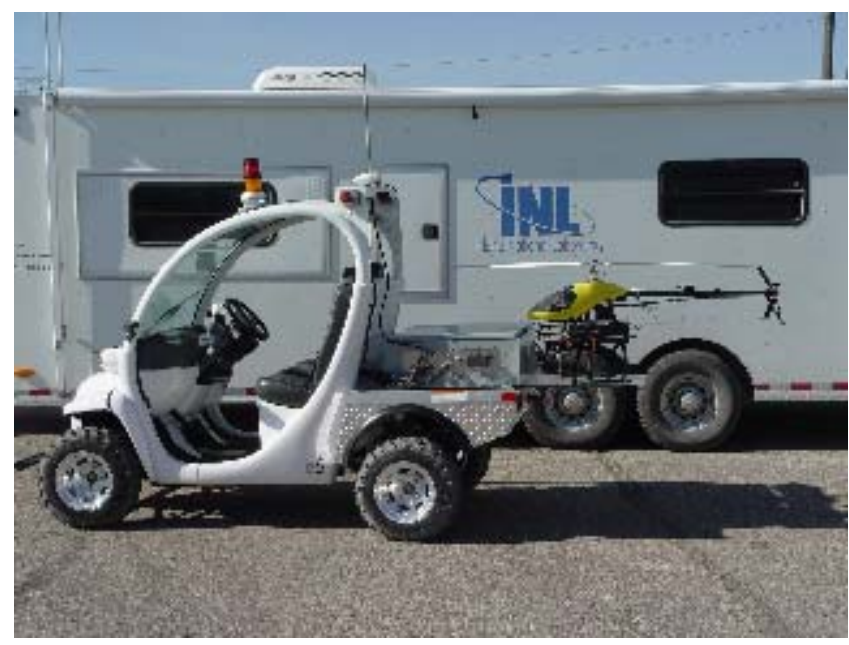

Figure 9. The E- RINO developed by Autonomous Solutions

\subsection{Common Operating Picture}

In order to better understand the situation as it unfolded, a common operating picture was developed. The goal of this effort was not necessarily to display all possible sources and sets of information, but rather to support an operator's dynamic visualization of the environment, the automatic correlation of various data sources, and the ability to task and use the various unmanned assets. The visualization of the operational scenario was accomplished by creating a virtual 3D environment that used static geo-referenced aerial imagery (either from satellite or previous air vehicle flights) as the back-drop for the visualization and tasking.

Imagery received from the unmanned air vehicles involved in the scenario was geo-referenced and illustrated by overlaying it on top of the original aerial imagery and the newer imagery. Figure 10 shows an illustration of how multiple unmanned air vehicle information can be viewed. 


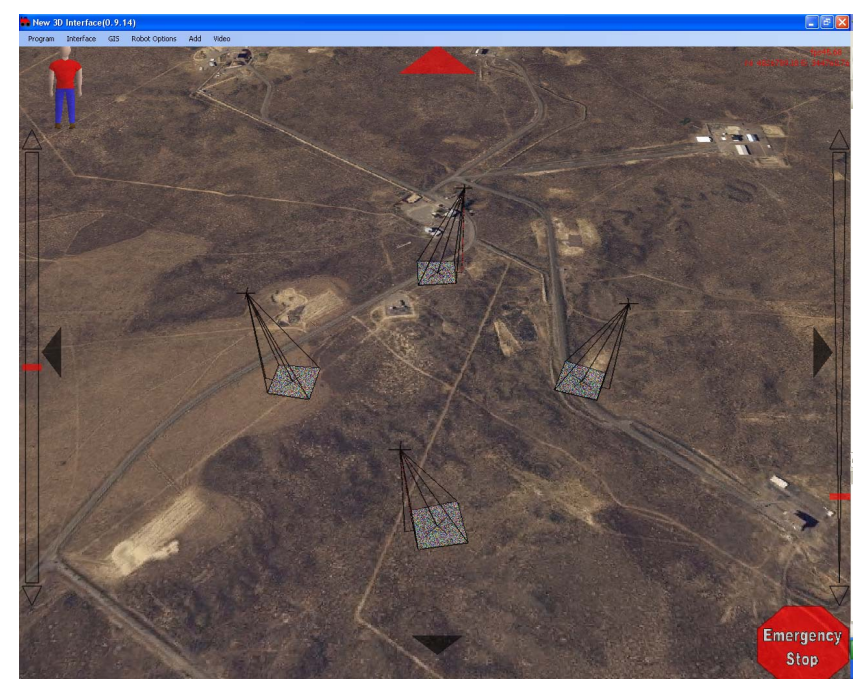

Figure 10. An illustration of the visualization of video streams from multiple unmanned air vehicles along with the relationships between the relative positions of the aircraft and where their cameras are focused.

The common operating picture is further designed to support simplified tasking of both unmanned air and ground vehicles. This is accomplished because the interface is geo-referenced, so when the operator selects places in the virtual environment they correlate directly to geo-referenced locations in the real-world and can therefore be used by the various unmanned vehicles and the gimbaled cameras.

\section{EXPERIMENT}

The scenario was designed as follows: Ground sensors would detect the presence of intruders and send messages to the command center. A high altitude UAV that is tasked for persistent surveillance is directed to classify the threat. If a threat is identified, the UAV estimates the ground track of the intruders and tasks several low altitude first responder air vehicles which are responsible for tracking the intruders and obtaining high resolution imagery of the intruders. Upon identification of the likely path of approach, a medium sized unmanned ground vehicle is dispatched to optimally intercept the intruders utilizing the prior site knowledge based path planning. Upon arrival, this UGV is used to verify the threat and possibly engage the intruders. Furthermore, an unmanned rotary craft is tasked to intercept and neutralize hostile forces as well as confirm results via helicopter-borne imagery. When the threat is neutralized, the air and ground vehicles return to the command center.

The invading force was represented by the E-RINO and was wirelessly driven to a place of intercept. The T-15 while performing persistent surveillance identified the target and began auto-tracking its movement. Information about the position of the invading robot was sent to the first responder air vehicles and to the RINO. The RINO used the a priori planned paths to determine a route that would lead to an intercept course based on the probable choice of paths of the invading robot. The first responder air vehicles provided eyes on the target and an electric powered rotary craft was dispatched to neutralize the target. Upon visual confirmation of the target with the rotary craft, the invading robot was assumed neutralized. Figure 11 shows the common operating picture when the ground and air vehicles were in-route (left side) and upon arrival near the target (right side). 


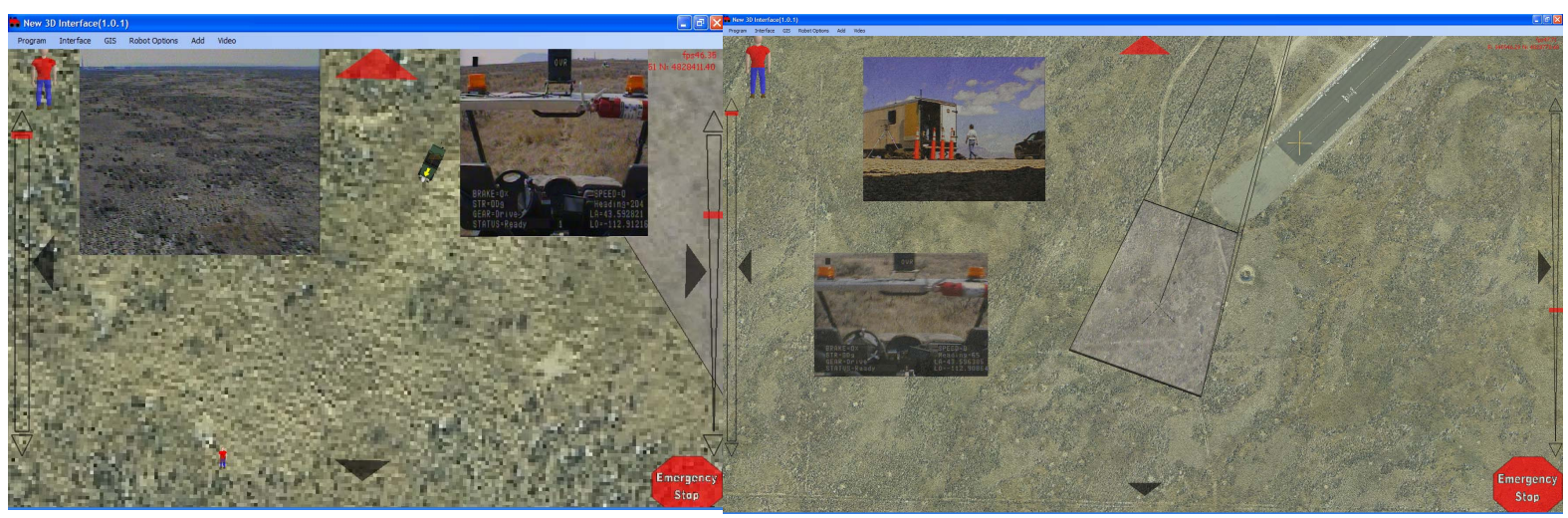

Figure 11. Snapshots of the common operating picture during the exercise.

\section{CONCLUSION}

The work described in this paper illustrates the possibility of using multiple unmanned vehicles to perform a site security task. The approach used multiple unmanned air vehicles including high-altitude and low-altitude fixed-wings as well as rotary-craft, medium sized ATV-based unmanned ground vehicles, and prior knowledge about the environment to ascertain the location of invading forces and intercept them. A common operating picture was developed to intuitively illustrate to the operator the present location of all assets, and once identified, to track the movement of the incoming invaders. The common operating picture showed a way to provide video from various sources in a manner that illustrates the positional relationships between all entities.

From this demonstration, there are many lessons learned. Primarily, when geo-referencing real-time imagery, the correlation was helpful but needs to be more accurate. In the experiment, it served to provide the operator with a correct assessment of the general location of activity, although it was not very precise. This did, however, enable the operator to task the UGV in the right direction. Multiple UAVs were effective at tracking the target from high and low altitudes, however, the tracking algorithms could be improved because they often lost track of the vehicle if the video was noisy or distorted. It should be noted, that the tracking was very helpful in that it reduced the effort required by the operator to keep the camera near the target.

Additionally, there were some issues with the prior path-planning that made correlation with the actual vehicle and the planned path difficult to follow. For example, sometimes the robot would be tasked right down the road, but would travel about 2 meters to the side of the road. This correlation seemed to fluctuate based on fluctuations in GPS. A better solution may be to use the prior path plan as the basis for dynamic planning depending on the robot's current view of the environment. Another approach might be to manually shift the robot's perception of its location to match the georeferenced location of the map. Future work will have to address these questions.

\section{Acknowledgment}

The authors would like to recognize the BYU Magicc lab, Solution Engineering and Cloud Cap Technologies, and Perry Kaveros for their efforts and support for the UAV portion of this demonstration. Furthermore, Autonomous Solutions and Kairos Autonomi provided exceptional assistance on the development and field deployment of the robotic platforms used in the experiment. This work was supported by the U.S. Department of Defense, under DOE Idaho Operations Office Contract DE-AC07-05ID14517. 


\section{References}

[1] Matthew O. Anderson, Mark D. McKay, Derek C. Wadsworth, "Intelligent Unmanned Vehicle Systems Suitable for Individual or Cooperative Missions," SPIE Unmanned Systems IX, Orlando, FL, April 2007

[2] Jeffrey B. Saunders, Brandon Call, Andrew Curtis, Randal W. Beard, Timothy W. McLain, "Static and Dynamic Obstacle Avoidance in Miniature Air Vehicles," AIAA Infotech@Aerospace Conference, Arlington, VA, September, 2005, Paper no. AIAA-2005-6950.

[3] Stephen Griffiths, Jeff Saunders, Andrew Curtis, D. Blake Barber, Timothy W. McLain, Randal W. Beard, "Maximizing miniature aerial vehicles," IEEE Robotics and Automation Magazine, vol. 13, no. 3, 2006 pp. 34-43.

[4] Timothy W. McLain, Randal W. Beard, "Coordination Variables, Coordination Functions, and Cooperative Timing Missions," AIAA Journal of Guidance, Control, \& Dynamics, vol. 28, no. 1., January, 2005, pp. 150-161.

[5] D. Blake Barber, Joshua D. Redding, Timothy W. McLain, Randal W. Beard, Clark N. Taylor, "Vision-based Target Geo-location using a Fixed-wing Miniature Air Vehicle," Journal of Intelligent and Robotic Systems, vol. 47, no. 4, December, 2006, pp. 361-382.

[6] Miles C. Walton, Douglas A. Few, David J. Bruemmer, "Improved Human-robot Teaming Through Facilitated Initiative," Proceedings of the 15th IEEE International Symposium on Robot and Human Interactive Communication, Hatfield, United Kingdom, September 2006.

\section{Copyright Statement}

The submitted manuscript has been authored by a contractor of the U.S. Government under DOE Contract DE-AC0705ID14517. Accordingly, the U.S. Government retains a nonexclusive, royalty-free license to publish or reproduce the published form of this contribution, or allow others to do so, for U.S. Government purposes. 